\title{
A Dynamic Programming Approach to Sustainability
}

\author{
Rani G. Selvanathan ${ }^{1}$ \\ ${ }^{1}$ Department of Management, West Chester University, West Chester PA, USA \\ Correspondence: Rani G. Selvanathan, 125 Scarlet Drive, Conshohocken, PA 19428, USA. Tel: 1-610-504-2128. \\ E-mail: Rselvanathan@wcupa.edu
}

Received: November 28, 2014 Accepted: January 19, 2015 Online Published: February 20, 2015

doi:10.5539/jms.v5n1p1 URL: http://dx.doi.org/10.5539/jms.v5n1p1

\begin{abstract}
This paper proposes to formulate the sustainability management problem as a multi-objective or goal programming problem, in order that the level of activities engaged by a corporation meets the three objectives of economic, environmental, and social obligation. Further, noted in the paper is the fact that sustainability optimization does not end within one time period but is an on-going activity. The paper further links the dynamic programming concept to model this on-going nature of sustainability measurements. Optimal levels of activities resulting in a given triple bottom line situation are linked to the future levels of the same activities.
\end{abstract}

Keywords: mathematical formulation, optimizing sustainability

\section{Sustainability and Triple Bottom Line}

A corporation's responsibility should not rest with economic growth alone but extend over to cover other environmental elements it influences. The external elements affecting a corporation can be classified broadly under two categories: environmental and social. Current thinking in the field of management reflects an approach that outlines the objectives that corporations hope to achieve. Actions of a corporation should therefore assure at least a minimum performance on the three fronts: economic (Profits), environment (Planet), and social (People). This concept has been referred to as a Triple Bottom Line (TBL).

Introduced by John Elkington in 1994, current usage of triple bottom line is a measure of a company's economic value along with its social and environmental responsibilities. Interest in triple bottom line has seen a consistent growth ever since its introduction. Used in varying ways, the concept of TBL has its applications in organizations, both for profit and not for profit, government, and regional development programs. The ways to assess a company's economic value is more quantifiable as compared to its social and environmental responsibilities and the three put together do not have a common unit of measurement. The benefits of Triple Bottom Line approach has been widely discussed by Willard, B. (2002). The concept of Triple Bottom Line and the problematic application associated with it arise from a) lack of integration among the three factors considered in the TBL and b) lack of well-defined relationships between the factors of TBL. Critiques of the approach Norman et al. (2004) question the possibility of managers and investors, while using the TBL, being distracted from achieving more effective social and environmental objectives.

International Council for Local Environmental Initiatives (ICLEI) founded in 1990 and United Nations in 2007 saw a need for a unified approach to assure the triple bottom line by both public and private sector corporations. It is to be noted that the level of importance given to the three fronts of economic, environmental and social could be different for public and private sector corporation and among different industries with the sectors.

The overall objective of sustainability management is to choose the levels of activities engaged in by a corporation such that:

a) The profits generated or the costs incurred by the corporation are maximized/minimized thereby assuring economic sustainability.

b) The impact of the activities on the environment is minimized, thus maintaining environmental sustainability, and

c) The positive effects on the society resulting from the activities pursued is maximized thereby assuring an acceptable level of social sustainability.

This paper proposes to formulate the sustainability management problem as a multiple-objective or goal 
programming problem wherein the level of every major activity undertaken by a business/corporation affects the three facets of sustainability, i.e. economic, social, and environmental of the institution, public or private. The objective of the institution therefore is to see that all the three facets of sustainability are met. Further, it is to be noted that sustainability optimization does not end within one time period, but is rather an on-going process. The importance of treating sustainability management issues on a continuous rather than a discrete time scale was discussed by Gladwin et al. (1995) where sustainability is seen as evolving with space, time, and component parts of the manifest world. Levels of activities engaged in over a given time period will dictate the levels of TBL. This consequently influences the level of activities to be chosen over the following time period and beyond. The study also proposes to expand the multi-objective programming formulation to a dynamic programming problem in keeping with the reasoning that sustainability does not end with a single time period but is comprised of a series of multi-objective programming decisions encompassing several periods in the future. Let us consider a sustainability management problem to cover $(n+1)$ periods into the future. Each stage $i, i=1 \ldots(n+1)$ represents a multi-objective/goal programming formulation of the problem. The concept of the entire formulation can be represented as in Figure 1.

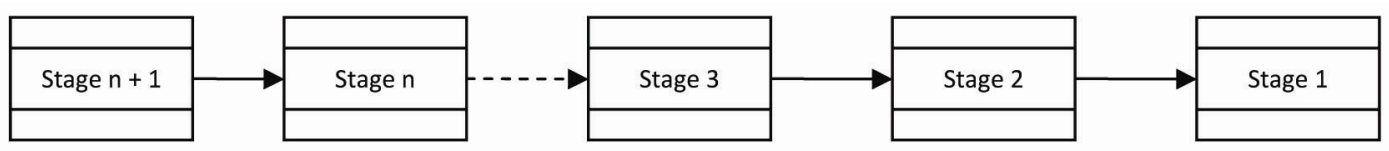

Figure 1. An $(\mathrm{n}+1)$ Stage Dynamic Programing Problem

Figure 2 illustrates in a greater detail, the functioning of a single stage (i).

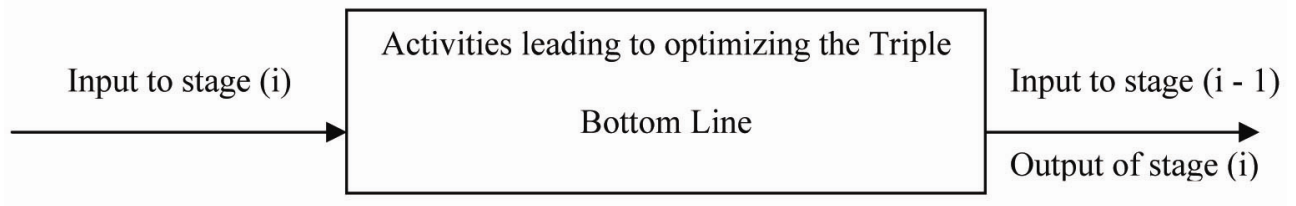

Stage (i), Goal Programming (i)

Figure 2. Sustainability Management over a Single Stage

\subsection{Economic Sustainability}

The economic performance of an organization can be measured by numerous indicators like, return on investment, productivity, market share, profits generated, to name a few, in addition to several other factors like profitability, sales growth, and market share Narasimhan and Kim (2002) by a certain course of action taken by the organization. They can be numbered as ESI-1, ESI-2 ..... Etc, where ESI-i represents an Economic Sustainability indicator generated as a result of activity $i$.

\subsection{Social Sustainability}

The corporate social responsibility (CSR) of an organization focuses on its public acts of functioning as a good citizen. It is commonly accepted by well published literature that social performance, CSR, has two aspects, internal and external. The internal aspect highlights employee well-being and equity as is evidenced by the works of Brown (1996); Hanna et al. (2000); Daily and Huang (2001); Vachon and Mao (2008); Pagell and Gobeli (2009) and Pullman et al. (2009). The external aspect is related to several community performance indicators such as corporate philanthropic commitment in studies by Montabon et al. (2007); Carter (2004); Vachon and Mao (2008); Pagell and Gobeli (2009); Pullman et al. (2009) and Jacobs et al. (2010). The decisions made by an organization in order for it to be socially sustainable are highly subjective in nature. Several indicators can be used to assess the organization's performance under this category. These indicators should in general start from the current desired position of the organization, and following the direction headed by the organization, will determine the extent of deviation from the social sustainability goal. Whatever the indicators may be, they all should be, without exception, measurable, relevant, understandable, reliable, and timely.

Practically all indicators of social sustainability consider the industry or the country as a whole. The choice of a decision alternative, made by a single organization or business is likely to have a very small/negligible impact on 
an economy's social sustainability numbers. Meeting sustainability goals becomes an option for any individual business concerned with its Corporate Social Responsibility (CSR). We propose, in this study, to measure the social sustainability of a business using three distinct indicators as indicated below;

- Percentage of new jobs created over a given period. This number, if positive, will indicate that a given action taken by the business has resulted in job creation, and if negative will indicate a loss of jobs due to reasons like outsourcing or restructuring, to name a few. It will measure therefore the impact of the business decisions on the business's social environment.

- Number of complaints regarding the product or service offered by the business.

- Ecological footprint which measures the amount of natural resources used by the business compared with the available natural resources, and can be represented by SSI-1, SSI-2, ...etc. Wackernagel (1991)

There exists numerous other ways of measuring social sustainability as discussed by Hutchins (2008).

\subsection{Environmental Sustainability}

Proposed by President Richard M. Nixon, the United States Environmental Protection Agency (EPA) began its operation on December 2, 1970. The agency is responsible for enforcing accepted standards or developing new standards under several environmental laws using its powers of administering fines, and sanctions to name a few. It also works with industries so as to maintain an acceptable level of pollution prevention and energy conservation measures. Environmental criteria can be established along two aspects, minimizing the negative impact of environmental waste and the corresponding risks involved and promoting more efficient use of resources. Works of Zhu and Sarkis (2004); Hervani et al. (2005); Matos and Hall (2007); Jacobs et al. (2010) address this particular issue. Over 40 Acts that support EPA in its role to assure safeguards for the environment in areas of protection of air, water, land, endangered species, and disposal of hazardous waste are in place.

Another important, measurable factor that could be treated as an environmental sustainability constraint is the required adherence to Kyoto Protocol (2013) regarding carbon trading. The protocol addresses the "green-house" emissions by 2012 compared to the baseline of 1990, and recommends a world target of $5.2 \%$. it is to be noted that the target is individualized by country with European Union set at $8 \%$ and the U.S. at $7 \%$ for example. Whereas the protocol establishes the measures for countries, the same can be applied to individual organizations. This would constitute an environmental sustainability constraint an organization could face, satisfaction of which would undermine its profitability. Some of the measurable factors of assuring environmental sustainability are listed below:

Reliance of an organization's activities on fossil fuel could be yet another measurable constraint assuring environmental sustainability.

Working in conjunction with various industries the EPA works on several of the following programs which are industry specific:

Energy Star: Foster energy efficiency.

Safer Detergent Stewardship Initiative: Requires the use of surfactants which have minimum impact both on fresh and sea water aquatic life.

Fuel Economy: Measures the total volume of carbon captured from the exhausts.

Air Quality: Assesses, using mathematical models, pollution control strategies and the overall impact of the pollution sources.

Oil Pollution: Provides guidelines to store, transport, sell, and dispose of oil related products.

WaterSense: Encourages the efficient use of water in the United States.

Drinking Water: Measures contamination of drinking water and monitors the work to have a contaminant-free drinking water.

Research Vessels: Measures and monitors the off-shore dredging process to dispose of waste materials.

It is to be noted that not all Acts and measures listed above, though not exhaustive, are applicable to all industries, and only relevant acts need to be retained depending in the type of business under consideration.

\section{Mathematical Formulation}

A typical business/corporation/industry has three major obligations to fulfill. These obligations can be represented by sustainability in the area of economics, environment, and social. The three sustainability functions depend on the level of activity that is planned to be carried out. The level of activity can be measured in terms of money spent 
them. It has been observed that money spent on improving profitability can negatively affect the performance in the environmental and social areas. Let us assume that there are " $\mathrm{k}$ " activities that are being planned over a time horizon of $\mathrm{n}$ (years). Every year of the time horizon is called a stage.

Let

$X_{i, j}$ represent the level of activity $i$ in stage $j$

For all $\mathrm{i}=1,2 . . . \mathrm{k}$ and

$$
\mathrm{j}=1,2 \ldots n \text {. }
$$

$P_{i, j}$ represent the returns (profit/loss) resulting from an activity $X_{i, j}$

$E_{i, j}$ represent the environmental impact resulting from the activity $X_{i, j}$ and

$S_{i, j}$ represent the social impact resulting from the activity $X_{i, j}$.

The overall Profit Function obtained by performing all the "k" activities throughout the " $n$ " stages is given by:

$$
\text { Economic Function }=\sum \sum \mathrm{P}_{\mathrm{i}, \mathrm{j}} \mathrm{X}_{\mathrm{i}, \mathrm{j}}
$$

The environmental and social functions can be defined in a similar way as:

$$
\begin{gathered}
\text { Environmental Function }=\sum \sum \mathrm{E}_{\mathrm{i}, \mathrm{j}} \mathrm{X}_{\mathrm{i}, \mathrm{j}} \\
\quad \text { Social Function }=\sum \sum \mathrm{S}_{\mathrm{i}, \mathrm{j}} \mathrm{X}_{\mathrm{i}, \mathrm{j}}
\end{gathered}
$$

Where the summation is carried out over values of $i=1, \ldots k$ and $j=1 \ldots n$

The level of activities in any given stage is limited by the budget allocated to the entire set of "k" activities at each of the " $\mathrm{j}$ " stages. If $\mathrm{B}_{\mathrm{j}}$ were the budget allocated to the activities, this translates into:

$$
\text { Budget Limitation }=\sum X_{i, j} \leqq B_{j}
$$

The fact that the levels of activities $\mathrm{X}_{\mathrm{i}, \mathrm{j}}$ cannot be negative and can be formulated as:

$$
\text { All } X_{i, j} \geqq 0
$$

It is to be noted that there can be more than one economic, environmental, and social functions depending on the type and relevancy of the respective indicators chosen.

The three functions defined above in equations (1), (2), and (3) along with the budget limitation in equation (4) and the non-negativity feature of the activity variables represented by equation (5), form the driving force of the formulation of sustainability management problem in any one given stage.

Multiple formulations of sustainability management problem are given below:

\subsection{Single Stage Problem as a Linear Programming Problem}

Given a single stage, the economic function pertaining to the stage in question can be treated as an objective function to be maximized with minimum requirements being placed on environmental and social functions acting as constraints along with the budget restrictions. We therefore have,

$$
\begin{gathered}
\operatorname{Max} \sum \mathrm{P}_{\mathrm{i}} \mathrm{X}_{\mathrm{i}} \\
\text { Subject to } \sum \mathrm{E}_{\mathrm{i}} \mathrm{X}_{\mathrm{i}} \geqq \mathrm{E} \\
\sum \mathrm{S}_{\mathrm{i}} \mathrm{X}_{\mathrm{i}} \geqq \mathrm{S} \\
\sum \mathrm{X}_{\mathrm{i}} \leqq \mathrm{B} \\
\text { All } \mathrm{X}_{\mathrm{i}} \geqq 0 \text { for all } \mathrm{i}=1,2 \ldots \mathrm{k}
\end{gathered}
$$

Where $\mathrm{E}$, and $\mathrm{S}$ represent the minimum level of environmental and social criteria that are to be met. Depending on the nature of the organization, we can have either the environmental or the social function as the objective, with the other two functions taking the place of constraints along with the budget constraint and the non-negativity restrictions.

It is to be noted that the logic of optimizing in a linear programming problem, as given by equations (6)..... (10), gives priority to maximize/minimize the objective function while minimally satisfying the constraints. Possibility of having no common region over which both the constraints are valid exists, thus giving rise to an infeasible solution

\subsection{Single-Stage Problem as a Multi-Objective or a Goal Programming Problem}

With the increasing awareness by the organizations of the fact that profit should not be their over riding goal and 
that an increase of which ought not to be occurring at the expense of the organizations' commitment to its environment and to the society it is a part of, having a sole objective of profit maximization does not correctly handle the problem. Similarly, maximizing social function should not be the unique, overpowering goal for it may put the economic viability of the organization in jeopardy. Thus the problem of sustainable management is more amenable to be treated as a problem with multiple objectives.

Working under the general premise of linear programming, the multi-objective programming involves solving of a series of linear programming problems in which there could be more than one objective present. For an organization, the three main criteria that govern its functioning are economic (profit), environment (planet), and social (people). It is to be noted here that the criteria could often be contradictory and work against each other.

The formulation of sustainability management problem as a multiple-objective or goal programming problem begins with setting a priority level, with 1 representing the goal with the highest priority, for each of the decision criteria. The priority levels themselves are changeable depending whether the organization belongs to public or private sector. A goal having priority level lis considered the primary goal whereas the other goals are considered secondary. The primary and secondary goals are known as preemptive goals in goal programming to represent the fact the decision maker will not compromise the level of attainment of a goal with a higher priority to satisfy the goal of a lower priority.

Since the goal, irrespective of its priority, may or may not be achieved, we define two additional variables, called deviational variables, for reach priority level. The role of the deviational variables is to allow a range around which the desired value of the objective function will vary.

Let

$\mathrm{d}_{1}^{+}$be the amount by which the actual profit exceeds the desired profits and

$\mathrm{d}_{1}^{-}$be the amount of shortfall in the actual profit from the desired value

The deviational variables for environmental objective $\left(\mathrm{d}_{2}{ }^{+}, \mathrm{d}_{2}{ }^{-}\right)$and for social objective $\left(\mathrm{d}_{3}{ }^{+}, \mathrm{d}_{3}{ }^{-}\right)$can be defined in a similar manner.

Goal programming solves a number of linear programming problems in order of their priority ranking, the number of problems solved depending on the number of goals in the problem. For sustainability management, if the profit, environmental, and social functions are measured in a unique way under the respective category, the goal programming problem will be solving three linear programs.

Step 1.

Formulation of the profit maximization, if prioritized as 1 is given below:

In the profit function, any positive deviation would be most desired, and a negative deviation needs to be avoided. This leads us to the formulation:

$$
\operatorname{Min} \mathrm{d}_{1}^{-}
$$

Subject to

$$
\begin{array}{rrr}
\sum \mathrm{X}_{\mathrm{i}} \leqq \mathrm{B} & \text { Budget limitation } \\
\sum \mathrm{P}_{\mathrm{i}} \mathrm{X}_{\mathrm{i}}-\mathrm{d}_{1}{ }^{+}+\mathrm{d}_{1}{ }^{-} \geqq \mathrm{P} & \text { Goal priority } 1 \\
\sum \mathrm{E}_{\mathrm{i}} \mathrm{X}_{\mathrm{i}}-\mathrm{d}_{2}{ }^{+}+\mathrm{d}_{2}{ }^{-} \geqq \mathrm{E} & \text { Goal priority } 2 \\
\sum \mathrm{S}_{\mathrm{i}} \mathrm{X}_{\mathrm{i}}-\mathrm{d}_{3}{ }^{+}+\mathrm{d}_{3}^{-} \geqq \mathrm{S} & \text { Goal priorit } 3 \\
\text { All } \mathrm{X}_{\mathrm{i}}, \mathrm{d}_{1}^{+}, \mathrm{d}_{1}{ }^{-}, \mathrm{d}_{2}{ }^{+}, \mathrm{d}_{2}{ }^{-}, \mathrm{d}_{3}{ }^{+}, \mathrm{d}_{3}^{-} \geqq 0 \text { for all } \mathrm{i}=1,2 \ldots \mathrm{k}
\end{array}
$$

Step 2

The problem formulation at the next lower level 2 is:

$$
\text { Min } \mathrm{d}_{2}{ }^{-}
$$

Subject to:

$$
\begin{array}{cr}
\sum X_{\mathrm{i}} \leqq \mathrm{B} & \text { Budget limitation } \\
\sum \mathrm{P}_{\mathrm{i}} \mathrm{X}_{\mathrm{i}}-\mathrm{d}_{1}^{+}+\mathrm{d}_{1}^{-} \geqq \mathrm{P} & \text { Goal priority 1 } \\
\sum \mathrm{E}_{\mathrm{i}} \mathrm{X}_{\mathrm{i}}-\mathrm{d}_{2}^{+}+\mathrm{d}_{2}^{-} \geqq \mathrm{E} & \text { Goal priority 2 }
\end{array}
$$




$$
\begin{aligned}
& \sum \mathrm{S}_{\mathrm{i}} \mathrm{X}_{\mathrm{i}}-\mathrm{d}_{3}{ }^{+}+\mathrm{d}_{3}{ }^{-} \geqq \mathrm{S} \quad \text { Goal priority } 3 \\
& \mathrm{~d}_{1}^{+}=0 \quad \text { Goal of P1 level is maintained } \\
& \text { All } X_{\mathrm{i}}, \mathrm{d}_{1}{ }^{+}, \mathrm{d}_{1}{ }^{-}, \mathrm{d}_{2}{ }^{+}, \mathrm{d}_{2}{ }^{-}, \mathrm{d}_{3}{ }^{+}, \mathrm{d}_{3}{ }^{-} \geqq 0 \text { for all } \mathrm{i}=1,2 \ldots \mathrm{k}
\end{aligned}
$$

Step 3.

The problem for priority level 3 can be developed in the same way by replacing the objective function in Step 2 by $\mathrm{d}_{3}{ }^{+}$and adding yet another constraint that assures that the goals achieved in levels P1 and P2 are maintained as follows:

$$
\mathrm{d}_{3}^{+}=0
$$

The general formulation of a sustainability management problem as a multi-objective programming problem can be given as:

$$
\operatorname{Min} \operatorname{PL} 1\left(\mathrm{~d}_{1}{ }^{+}\right)+\operatorname{PL} 2\left(\mathrm{~d}_{2}^{+}\right)+\operatorname{PL} 3\left(\mathrm{~d}_{3}{ }^{+}\right)
$$

PL1, PL2, PL3 etc. Do not have any numerical value but merely indicate the priority level of the deviation variable Subject to:

$$
\begin{array}{cl}
\sum \mathrm{X}_{\mathrm{i}} \leqq \mathrm{B} & \multicolumn{1}{c}{\text { Budget limitation }} \\
\sum \mathrm{P}_{\mathrm{i}} \mathrm{X}_{\mathrm{i}}-\mathrm{d}_{1}^{+}+\mathrm{d}_{1}^{-}=\mathrm{P} & \text { Goal priority } 1 \\
\sum \mathrm{E}_{\mathrm{i}} \mathrm{X}_{\mathrm{i}}-\mathrm{d}_{2}^{+}+\mathrm{d}_{2}^{-}=\mathrm{E} & \text { Goal priority } 2 \\
\sum \mathrm{S}_{\mathrm{i}} \mathrm{X}_{\mathrm{i}}-\mathrm{d}_{3}^{+}+\mathrm{d}_{3}^{-}=\mathrm{S} & \text { Goal priority } 3
\end{array}
$$

It is to be noted that there could be multiple goals at the same priority level. Weights can be assigned to the goals at the same priority level. For example let us consider two social sustainability goals, denoted as A and B to be met at level 3 leading to defining a new set of deviational variables, $\mathrm{d}_{3}{ }^{+}, \mathrm{d}_{3}{ }^{-}, \mathrm{d}_{4}{ }^{+}$, and $\mathrm{d}_{4}{ }^{-}$. For illustration purposes, if achieving goal $\mathrm{A}$ is twice as important as achieving goal $\mathrm{B}$, the objective function in equation (25) would be replaced by

$$
\text { Min PL1 }\left(\mathrm{d}_{1}{ }^{+}\right)+\operatorname{PL} 2\left(\mathrm{~d}_{2}{ }^{+}\right)+\operatorname{PL} 3\left(2 \mathrm{~d}_{3}{ }^{+}\right)+\operatorname{PL} 3\left(\mathrm{~d}_{4}{ }^{+}\right)
$$

And constraint (29) would be replaced by

$$
\begin{aligned}
& \sum \mathrm{S}_{\mathrm{i}} \mathrm{X}_{\mathrm{i}}-\mathrm{d}_{3}{ }^{+}+\mathrm{d}_{3}{ }^{-}=\mathrm{A} \\
& \sum \mathrm{S}_{\mathrm{i}} \mathrm{X}_{\mathrm{i}}-\mathrm{d}_{3}{ }^{+}+\mathrm{d}_{3}{ }^{-}=\mathrm{B}
\end{aligned}
$$

Along with $\mathrm{d} 4+$, and $\mathrm{d} 4$ - requiring to be non-negative added to constraint (30).

The same reasoning can be extended to formulate a situation wherein the goal to achieve at each priority level is not just a single number but can assume a value over a given range of values.

\subsection{Multi-Stage Problem as a Dynamic Programming Problem}

Managing economic, environmental, and social sustainability over a long period of time is a daunting task. Dynamic programming can be used to formulate such a problem. Dynamic programming is a series of smaller optimization models linked to each other in such a way that a systematic solving of the smaller problems leads to solving the larger problem as a whole.

Proposed by Richard Bellman in 1952, the "Principle of Optimality" provides the starting point to the way of solving a dynamic programming problem. The Principle of Optimality can be stated as "An optimal policy has the property that, whatever the current state and decision, the remaining decisions must constitute an optimal policy with regards to the state resulting from the current decision". More detailed discussion on the concept of dynamic programming can be found in Bellman (1965).

Dynamic programming is more of a concept that gives an approach to solve larger problems rather than provide a fixed solution procedure or algorithm. The concept itself is highly adaptable to a variety of practical situations like water resources optimization by Heidari et al. (2005); Morin et al. (1971); Mawer et al. (1974); Murray et al. (1979); and Tauxe et al. (1982). Geiger et al. (1995); Dadebo (1995) applied dynamic programming to chemical engineering problems. Dynamic programming has also been applied to sequencing problems by Held et al. (1962). Due to the ability of addressing practical situations wherein decisions and corresponding actions are done in a sequential manner with consequences of decisions made at a particular point in time affecting subsequent 
decisions make it amenable to study the problem of sustainability management. Over a 10-year period, for example, this formulation can be seen as sustainability management problem over 10 stages, with each stage representing one year. In addition to the stages, which specify how the problem looks at different points in time, there are state variables associated with each stage. In a typical sustainability management situation, the current values of the economic, environmental, and social functions constitute the state variables. It is to be noted that the computational effort and cost increase dramatically as the number of states increase beyond 3 .

We propose to approach the sustainability management problem through a recursive process using forward induction, where stage 1 (year 1) problem is solved to start with. Problems are solved one stage at a time by including one past year at a time until the entire planning period is covered.

The dynamic programming formulation begins with the following definitions:

Let us assume that the sustainability of an organization is to be assured over $\mathrm{N}$ years. Each year is considered as one stage in a dynamic programming formulation.

Let $\mathrm{s}_{\mathrm{n}}$ represent the state of the system while at stage (with $\mathrm{n}$ stages to go).

Let $d_{n}$ be the decision made at stage $n$, which along with $s_{n}$, will propel the system to the next stage (n-1).

The move from stage $\mathrm{n}$ to stage $(\mathrm{n}-1)$ is represented by $\mathrm{t}_{\mathrm{n}}$ known as "transition function" and can be represented by the equation

$$
\mathrm{s}_{\mathrm{n}-1}=\mathrm{t}_{\mathrm{n}}\left(\mathrm{s}_{\mathrm{n}}, \mathrm{d}_{\mathrm{n}}\right)
$$

Decision $d_{n}$ belongs to a set of possible decisions $D_{n}$, and produces a return of $f_{n}\left(d_{n}, s_{n}\right)$ which takes the system to the new stage of (n-1).

In case of sustainability management, the return is the optimum value of the objective function of a corresponding linear programming or goal programming problem as described earlier.

$\mathrm{v}_{\mathrm{n}}\left(\mathrm{s}_{\mathrm{n}}\right)$ be the optimal value of all prior decisions, given the problem is in state $\mathrm{s}_{\mathrm{n}}$ with (n-1) stages to go is given by;

$$
\begin{gathered}
\mathrm{v}_{\mathrm{n}-1}\left(\mathrm{~s}_{\mathrm{n}-1}\right)=\operatorname{Max} \mathrm{v}_{\mathrm{n}}\left(\mathrm{s}_{\mathrm{n}}\right)+\mathrm{f}_{\mathrm{n}}\left(\mathrm{d}_{\mathrm{n}}, \mathrm{s}_{\mathrm{n}}\right) \\
\text { Where } \mathrm{s}_{\mathrm{n}-1=}=\operatorname{tn}\left(\mathrm{d}_{\mathrm{n}}, \mathrm{s}_{\mathrm{n}}\right)
\end{gathered}
$$

The sustainability management problem, over a two-year period is represented in Figure 3., as earlier stated as

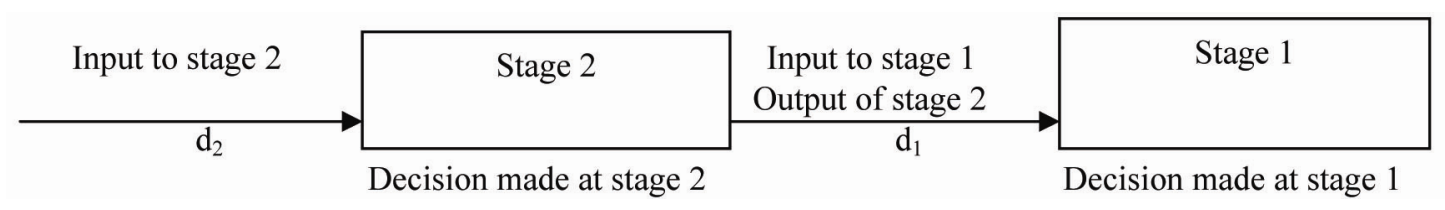

Figure 3. Two-Stage Sustainability Management Problem

\section{Conclusion}

The ways to assure social and environmental sustainability are numerous. Lack of common unit of measurement makes a corporation address the three factors of TBL independently of each other. The interaction between the factors of TBL is rarely investigated. It is to be noted that working towards social or environmental sustainability will have an effect on a corporation's economic sustainability.

This work therefore, attempts to link the three factors through its formulation as a linear and goal programming problem. It further stresses the need to consider, by means of the dynamic programming approach, the factors on an annual basis and reinforce the fact that pursuit of any one of the sustainability factor will come at the expense of the other two. Such study can help organizations determine the extent of influence a pursuit of social and environmental sustainability goals has on its economic performance. Organizations can therefore determine its level of involvement in assuring social and environmental sustainability for a desired level of economic performance.

It is to be noted that unlike Linear Programming problems, which can be solved in a preset algorithmic manner using any one of the existing computer software, Goal Programming and Dynamic Programming problems are contingent based and their formulation could vary from one problem to another. Computer software to solve the 
later mentioned programs are rare to find and difficult to implement. Solutions to these problems is however possible by solving a series of linear programming problems.

Solutions to problems with infinite stages and to those problems that requires the decision variables to be continuous are calculus based. Formulation of such categories of problems is possible but the practical applicability of the solution procedures can be a venue of future investigation.

\section{References}

Bellman, R. (1952). On the Theory of Dynamic Programming. Proceedings of the National Academy of Sciences. http://dx.doi.org/10.1073/pnas.38.8.716

Bellman, R., \& Kalaba, R. E. (1965). Dynamic programming and modern control theory.

Brown, V. A. (1996). Double or nothing: the changing relationship between the health of the biosphere and the health of the people. Proceedings of a Conference Held at the Australian National University, November 30-December 1, 1995. Nature and Society Forum, Canberra, pp. 59-67.

Carter, C. R. (2004). Purchasing and social responsibility: a replication and extension. Journal of Supply Chain Management, 40(4), 4-16. http://dx.doi.org/10.1111/j.1745-493X.2004.tb00175.x

Dadebo, S. A., \& McAuley, K. B. (1995). Dynamic optimization of constrained chemical engineering problems using dynamic programming. Computers \& Chemical Engineering. http://dx.doi.org/10.1016/0098-1354(94)00086-4

Daily, B. F., \& Huang, S. (2001). Achieving sustainability through attention to human resource factors in environmental management. International Journal of Operations and Production Management, 21(12), 1539-1552. http://dx.doi.org/10.1108/01443570110410892

Elkington, J. (1997). Cannibals with Forks: The Triple Bottom Line of the $21^{\text {st }}$ Century Business, Capstone.

Geiger, D. et al. (1995). Dynamic programming for detecting, tracking, and matching deformable contours. Institute of Electrical and Electronics Engineers Xplore, 17(3).

Gladwin, T. N., \& Krause, J. J. (1995). Shifting Paradigms for Sustainable Development: Implications for Management Theory and Practice. Academy of Management Review, 20(4), 874-907.

Hanna, M. D., \& Newman, W. R. et al. (2000). Linking operational and environmental improvement through employee involvement. International Journal of Operations \& Production Management, 20(2), 148-165. http://dx.doi.org/10.1108/01443570010304233

Heidari, M. et al. (1971). Discrete differential dynamic programing approach to water resources systems optimization.

Held, M., \& Karp, R. (1962). A Dynamic Programming Approach to Sequencing Problems. Journal of the Society for Industrial \& Applied Management (SIAM), 10, 196-210. http://dx.doi.org/10.1137/0110015

Hervani, A. A., \& Helms, M. M. et al. (2005). Performance Measurement for Green Supply Chain Management. Benchmarking: An International Journal, 12(4), 330-353. http://dx.doi.org/10.1108/14635770510609015

Hutchins, M. J., \& Sutherland, J. W. (2008). An exploration of measures of social sustainability and their application to supply chain decisions. Journal of Operations Management, (25), 998-1014.

Jacobs, J. F., \& Van de Poel, I. et al. (2010). Towards Safety and Sustainability by Design: Nano-sized TiO2 in Sunscreens Understanding Nanotechnology, U. Fiedeler et al. (Eds.) AKA Verlag Heidelberg.

Matos, S., \& Hall, J. (2007). Integrating sustainable development in the extended value chain: The case of life cycle assessment in the oil \& gas and agricultural biotechnology industries. Journal of Operations Management, 25, 1083-1102. http://dx.doi.org/10.1016/j.jom.2007.01.013

Mawer, P. A., \& Thorn, D. (1974). Improved dynamic programming procedures and their practical application to water resource systems. Water Resources Research. http://dx.doi.org/10.1029/WR010i002p00183

Montabon, F., \& Sroufe, R. P. et al. (2007). An Examination of Corporate Reporting, Environmental Manage-ment Practices and Firm Performance. Journal of Operations Management, (25), 998-1014. http://dx.doi.org/10.1016/j.jom.2006.10.003

Morin, T. L., \& Esogbue, A. (1971). Some efficient dynamic programming algorithms for the optimal sequencing and scheduling of water supply projects. Water Resources Research. http://dx.doi.org/10.1029/WR007i003p00479 
Murray, D. M., \& Yakowitz, S. J. (1979). Constrained differential dynamic programming and its application to multireservoir control. Water Resources Research. http://dx.doi.org/10.1029/WR015i005p01017

Narasimhan, R., \& Kim, S. W. (2002). Effect of supply chain integration on the relationship between diversification and performance: evidence from Japanese and Korean firms. Journal of Operations Management, 20(3), 303-323. http://dx.doi.org/10.1016/S0272-6963(02)00008-6

Norman, W., \& McDonald, C. (2004). Getting to the Bottom of "Triple Bottom Line”. Business Ethics Quarterly. http://dx.doi.org/10.5840/beq200414211

Pagell, M., \& Gobeli, D. (2009). How Plant Managers' Experiences and Attitudes Towards Sustainability Relate to Operational Performance. Production and Operations Management, 18(3), 278-299. http://dx.doi.org/10.1111/j.1937-5956.2009.01050.x

Pullman, M. E., \& Maloni, M. J. et al. (2009). Food for thought: Social versus environmental sustainability practices and performance outcomes. Journal of Supply Chain Management, 45(4), 38-54. http://dx.doi.org/10.1111/j.1745-493X.2009.03175.x

Tauxe, G. W., \& Inman, R. R. (1979). Multiobjective dynamic programming with application to a reservoir. Vachon, S., \& Mao, Z. (2008). Linking supply chain strength to sustainable development: a country-level analysis. Journal of Cleaner Production, 16, 1552-1560.

US Environmental Protection Agency. (2013). Retrieved from http://unfccc.int/kyoto_protocol/items/2830.php

Wackernagel, S. (1991). Land Use: Measuring a Community's Appropriated Carrying Capacity as an Indicator for Sustainability; and Using Appropriated Carrying Capacity as an Indicator, Measuring the Sustainability of a Community. Report I \& II to the UBC Task Force on Healthy and Sustainable Communities, Vancouver.

Willard, B. (2002). The Sustainability Advantage: Seven Business Case Benefits of a Triple Bottom Line. New Society Publishers.

Yakowitz, S. (1982) Dynamic programming applications in water resources research. Water Resources Research. http://dx.doi.org/10.1029/WR018i004p00673

Zhu, Q., \& Sarkis, J. (2004). Relationships between operational practices and performance among early adopters of green supply chain management practices in Chinese manufacturing enterprises. Journal of Operations Management, 22, 265-289. http://dx.doi.org/10.1016/j.jom.2004.01.005

\section{Copyrights}

Copyright for this article is retained by the author(s), with first publication rights granted to the journal.

This is an open-access article distributed under the terms and conditions of the Creative Commons Attribution license (http://creativecommons.org/licenses/by/3.0/). 